TRANSACTIONS OF THE

AMERICAN MATHEMATICAL SOCIETY

Volume 277, Number 1, May 1983

\title{
REAL-ANALYTIC SUBMANIFOLDS WHICH ARE LOCAL UNIQUENESS SETS FOR HOLOMORPHIC FUNCTIONS OF $\mathrm{C}^{3}$
}

BY

\author{
GARY A. HARRIS
}

\begin{abstract}
The following problem is considered. Given a real-analytic two-dimensional submanifold, $M$, of complex Euclidean three-space, are ambient holomorphic functions determined by their values on $M$ ? For a large class of submanifolds a necessary and sufficient condition is found for $M$ to be a local uniqueness set for holomorphic functions on complex three-space. Finally, the general problem is shown to be related to two-dimensional Nevanlinna theory.
\end{abstract}

0. We are interested in the problem of determining if a given real $m$-dimensional real-analytic submanifold, $M^{m}$, of complex Euclidean $n$-space, $\mathbf{C}^{n}$, is a local uniqueness set for holomorphic functions of $\mathbf{C}^{n}$.

Definition 0.1. A connected real-analytic submanifold $M^{m} \subset \mathbf{C}^{n}$ is a local uniqueness set for $\mathbf{C}^{n}$ at a point $p \in M^{m}$ provided, for any connected open subset $U \subset \mathbf{C}^{n}$ such that $p \in U$, and any holomorphic function $f: U \rightarrow \mathbf{C}$, if $\left.f\right|_{M \cap U} \equiv 0$ then $f \equiv 0$ on $U$.

If $M^{m}$ is a real-analytic C.R. submanifold of $\mathrm{C}^{n}$ it follows that $M$ is a local uniqueness set for $\mathbf{C}^{n}$ at $p \in M$ if and only if $M$ is generic in $\mathbf{C}^{n}$. A proof of this well-known result is given in $\S 1$, Proposition 1.4. If $M^{m}$ is not C.R., one can still define "generic": $M^{m}$ is generic if $M^{m}$ is generic away from its C.R. singular set. Because the C.R. singular set is nowhere dense in $M^{m}$ it follows that $M^{m}$ generic implies $M^{m}$ is a local uniqueness set for $\mathbf{C}^{n}$ at each point of $M^{m}$. Again a simple direct proof is given in Proposition 1.4. However, in the non-C.R. case the converse is not true. That is, a nongeneric submanifold can still be a local uniqueness set for $\mathrm{C}^{n}$ at some point on it. Indeed, as observed in [H-1 and H-2], the nongeneric submanifold $M^{2}$ given as Example 0.2 is a local uniqueness set for $\mathbf{C}^{3}$ at the origin.

EXAMPle 0.2. $M^{2} \equiv\left\{\left(x+i y,(x+i y) y,(x+i y) y e^{y}\right):(x, y) \in \mathbf{R}^{2}\right\} \subset \mathbf{C}^{3}$.

It is easy to see that any real-analytic real $(2 n-2)$-dimensional non-C.R. submanifold of $\mathbf{C}^{n}$ must be generic in $\mathbf{C}^{n}$ and hence is a local uniqueness set at each of its points. Therefore, Example 0.2 demonstrates the first possible situation in which a nongeneric submanifold can be a local uniqueness set for $\mathbf{C}^{n}$, namely, $M^{2} \subset \mathrm{C}^{3}$. It is this situation we will discuss.

Received by the editors August 14, 1981 and, in revised form, April 30, 1982.

1980 Mathematics Subject Classification. Primary 32C25; Secondary 13J05.

${ }^{1}$ Resarch partially supported by NSF Grant \#MCS 7800806 at Texas Tech University while the author was visiting at Rice University. 
Our first step is to place the problem in a more algebraic setting by a process which is valid for general $m$ and $n$. We effectively begin where [H-2] ends and apply the results and techniques of [H-2] to produce a canonical form, (1.10), for the special case $m=2$ and $n=3$. We are then able to apply a standard trick (see Remark 2.3) from Algebraic Geometry to construct a tool, Lemma 1.11, with which to study our problem. We then present several results and relevant examples which explain the problem in many cases. Indeed, the phenomenon as it occurs in Example 0.2 is completely characterized by our main result, Theorem 2.12, and by Remark 2.13. While Theorem 2.12 is not surprising, the remaining examples and results, in particular Theorem 2.23 and Remark 2.25, illustrate the subtle and difficult nature of the problem in the most general situation.

I am most grateful to Professor Reese Harvey for his helpful comments. I am also grateful to the referee for reminding me of the relevance of several nice general results from local analytic ring theory. Of particular relevance is the following result of Pierre Milman.

TheOrem (P. Milman [M]). Let $g: A \rightarrow B=K\{x\} / I$ be a polynomial homomorphism, where $K=\mathbf{C}$ or $K=\mathbf{R}$ and $I$ is a prime ideal generated by polynomials. Then rk $g=\operatorname{dim} A / \operatorname{ker} g$.

This Theorem immediately yields Remark 2.3(1). The referee further points out that using Chevalley's theorem and the proof of Milman's theorem one can obtain the analogous result for $g$ an algebraic homomorphism. In this manner one can obtain one direction of our Theorem 2.12, as well as Proposition 2.1 and Examples 2.4, 2.6 and 2.8 .

In the following discussion we need not appeal to these more general algebraic results because we are able to reduce our problem to a consideration of the particularly simple form $\phi$ in (1.10). Thus, applying a simple and direct technique, we are able to study the properties of $\operatorname{ker} \phi^{*}$ and obtain stronger results than those obtained from the general results indicated above.

1. Henceforth $M^{m}$ will denote a real $m$-dimensional real-analytic non-C.R. submanifold of $\mathbf{C}^{n}$. Without loss of generality we will assume the origin, 0 , belongs to the C.R. singular set of $M^{m}$, and we wish to determine if $M^{m}$ is a local uniqueness set for $\mathbf{C}^{n}$ at 0 . Because of the local nature of our problem we may assume there is an open neighborhood, $N$, of the origin in $\mathbf{R}^{m}$ and a real-analytic mapping $\psi \equiv$ $\left(\phi_{1}, \ldots, \phi_{n}\right): N \rightarrow \mathbf{C}^{n}$ such that the real rank of $\psi$ is $m$ (constant on $\left.N\right), \psi(0)=0$ and $M=\psi(N)$. We let $\Phi$ denote the complexification of $\psi$. Thus $\Phi$ is a holomorphic mapping from an open subset $U$ of $C^{m}$ to $C^{n}$ with $\Phi(0)=0$ and $M^{m} \subset \Phi(U)$. Let $\mathrm{C}_{m}$, respectively $\mathbf{C}_{n}$, denote the ring of convergent power series in $m$, respectively $n$, complex coordinates. Let $\Phi^{*}$ denote the homomorphism from $\mathbf{C}_{n}$ to $\mathbf{C}_{m}$ defined as follows: for $f \in \mathbf{C}_{n}, \Phi^{*} f \equiv f \circ \Phi$. It follows that $M$ is a uniqueness set for $\mathbf{C}_{n}$ at 0 if and only if $\operatorname{ker} \Phi^{*}=(0)$, i.e. $\Phi^{*}$ is injective $[\mathbf{H}-1, \S 6]$.

Definition 1.1. For an ideal $\mathfrak{A} \subset \mathrm{C}_{n}$ the corank of $\mathfrak{A}$, cg $\mathfrak{A}$, is defined by $\operatorname{cg} \mathfrak{A}=\operatorname{dim}_{\mathbf{C}} \mathfrak{A} / \mathfrak{A} \mathfrak{M}$. 
In Definition 1.1, $\mathfrak{M}$ denotes the maximal ideal in $\mathbf{C}_{n}$. An immediate consequence of the above discussion is

Proposition 1.2. $M^{m}$ is a local uniqueness set for $\mathbf{C}^{n}$ at 0 if and only if $\operatorname{cg}\left(\operatorname{ker} \Phi^{*}\right)=0$.

In [H-1] we see that $M^{m}$ is C.R. if and only if the complex Jacobian matrix of $\Phi$ has constant rank near 0 . If $M$ is not C.R. we define the rank $\Phi \equiv \operatorname{rank} \Phi^{*}$ to be the generic rank of the complex Jacobian matrix of $\Phi$. It follows that

$$
\operatorname{cg}\left(\operatorname{ker} \Phi^{*}\right) \leqslant n-\operatorname{rk} \Phi^{*} \text {. }
$$

We also see in [H-1] that $M^{m}$ is generic in $\mathbf{C}^{n}$ if and only if rk $\Phi^{*}=n$. Thus (1.3) and Proposition 1.2 yield

Proposition 1.4. If $\mathbf{M}^{m}$ is generic in $\mathbf{C}^{n}$ then $M^{m}$ is a local uniqueness set for $\mathbf{C}^{n}$ at 0 .

The above discussion is valid for general values of $m$ and $n$. However, we will henceforth restrict our attention to the special case $m=2$ and $n=3$. Given $M^{2}$ and a holomorphic mapping $\Phi$ associated with $M^{2}$ as above, the idea is to employ the technique used in [H-2], simplifying the form of $\Phi$ to a form for which we can

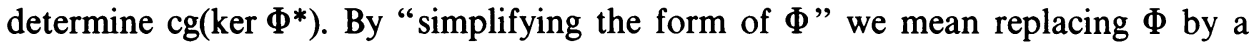
new holomorphic mapping, $\tilde{\Phi}$, with $\operatorname{cg}\left(\operatorname{ker} \Phi^{*}\right)=\operatorname{cg}\left(\operatorname{ker} \tilde{\Phi}^{*}\right)$.

REMARK 1.5. In the case $m=2$ and $n=3$ it follows from the construction of $\Phi$ that $M$ not C.R. implies rk $\Phi^{*}=2$. Therefore (1.3) yields either $\operatorname{cg}\left(\operatorname{ker} \Phi^{*}\right)=0$ or $\operatorname{cg}\left(\operatorname{ker} \Phi^{*}\right)=1$. Moreover, $m=2$ is the only case in $\mathbf{C}^{3}$ for which the problem is interesting.

We now proceed from where $[\mathbf{H}-2]$ concludes. Without loss of generality (see $[\mathbf{H}-2$, $\S 3$, Form IX]) we assume $\Phi$ is of the form

$$
\Phi=\left(Z, Z^{p} w^{m}, Z^{q} w^{n} U(Z, w)\right)
$$

where $1 \leqslant m<n<\infty, U \in \mathbf{C}_{2}$, and $U(0) \neq 0$. We have thus reduced the study of the local uniqueness set question for general real-analytic $M^{2} \subset \mathrm{C}^{3}$ to the study for $M^{2}$ of the special form

$$
M^{2}=\left(x+i y,(x+i y)^{p} y^{m},(x+i y)^{q} y^{n} U(x+i y, y)\right) .
$$

We should point out that for all examples in $\$ 2$ one can construct a submanifold $M^{2}$ from the given holomorphic mapping $\Phi$ in exactly the same way (1.7) is constructed from (1.6). $M^{2}$ will then be a local uniqueness set for $C^{3}$ if and only if $\operatorname{cg}\left(\operatorname{ker} \Phi^{*}\right)=0$. Observe that $\Phi$ associated with Example 0.2 is $\Phi=(Z, Z w, Z w \exp (w))$.

Biholomorphic coordinate changes in either the range or domain of $\Phi$ will yield a new mapping $\tilde{\Phi}$ with $\operatorname{cg}\left(\operatorname{ker} \Phi^{*}\right)=\operatorname{cg}\left(\operatorname{ker} \tilde{\Phi}^{*}\right)$. Thus we perform the coordinate change $Z \rightarrow Z, w \rightarrow Z+w$ in the domain of $\Phi$ and then subtract off the pure $Z$ terms in the second and third component functions by a range coordinate change. This yields a new mapping, again denoted by $\Phi$, of the form

$$
\Phi=\left(Z, Z^{p} w \sum_{v=1}^{m}\left(\begin{array}{c}
m \\
v
\end{array}\right) Z^{m-v} w^{v-1}, Z^{q} w\left[\sum_{v=1}^{n}\left(\begin{array}{l}
n \\
v
\end{array}\right) Z^{n-v} w^{v-1}\right] U(Z, w)\right)
$$


where $0 \leqslant p \leqslant q$ and $U \in \mathbf{C}_{2}$ is a unit but not necessarily the same as that in (1.6).

The mapping $H(Z, w) \equiv\left(Z, Z^{m+n-2} w\right)$ has generic rank 2 , hence $H^{*}: \mathbf{C}_{2} \rightarrow \mathbf{C}_{2}$ is injective and $\operatorname{cg}\left(\operatorname{ker}(\Phi \circ H)^{*}\right)=\operatorname{cg}\left(\operatorname{ker} \Phi^{*}\right)$. Thus we compose $\Phi$ with $H$ to get a new mapping, again denoted by $\Phi$,

$$
\Phi=\left(Z, Z^{p} w U_{1}, Z^{q} w U_{2}\right)
$$

where $1 \leqslant p \leqslant q$ are not necessarily the same as in (1.8) and $U_{1}, U_{2} \in \mathbf{C}_{2}$ are units. We now "absorb" $U_{1}$ into $W$ by a domain coordinate change to obtain the new mapping

$$
\Phi=\left(Z, Z^{p} w, Z^{q} w U(Z, w)\right)
$$

where $1 \leqslant p \leqslant q$ and $U$ is some unit in $\mathbf{C}_{2}$. After changing coordinates in the range if necessary, we may assume $U=1+w h$ for some $h \in \mathbf{C}_{2}$.

We conclude this section with

LEMMA 1.11. Suppose $\Phi$ is given in the form (1.10). Then $\operatorname{cg}\left(\operatorname{ker} \Phi^{*}\right)=1$ if and only if there exists $H \in \mathrm{C}_{3}$ such that $H \neq 0, X_{3} \mid H$, and

$$
H\left(Z_{1}, Z_{2}, Z_{1}^{q-p} Z_{2} U\left(Z_{1}, Z_{2} / Z_{1}^{p}\right)\right) \in \mathbf{C}_{2} \text {. }
$$

REMARK 1.12. Since $U$ is holomorphic in some neighborhood of 0 , the function $U\left(Z_{1}, Z_{2} / Z_{1}^{p}\right)$ is holomorphic on some open set $\Omega \subset \mathbf{C}_{2} \backslash\left\{Z_{1}=0\right\}$ with $0 \in \bar{\Omega}$. The existence of $H$ in Lemma 1.11 is a statement that $U\left(Z_{1}, Z_{2} / Z_{1}^{p}\right)$ is "extendable" across its singularity in some very general sense. From an algebraic viewpoint the existence of such $H$ is a condition on the relations among the various coefficients in the power series $U$. The results of $\$ 2$ provide better insight into the meaning of these conditions.

Proof of Lemma 1.11. $(\Leftarrow)$ Let $H \in \mathbf{C}_{3}$ be as hypothesized. Hence

$$
H\left(Z_{1}, Z_{2}, Z_{1}^{q-p} Z_{2} U\left(Z_{1}, Z_{2} / Z_{1}^{p}\right)\right) \equiv G\left(Z_{1}, Z_{2}\right)
$$

is in $\mathbf{C}_{2}$. Define $F \in \mathbf{C}_{3}$ by $F\left(X_{1}, X_{2}, X_{3}\right) \equiv H\left(X_{1}, X_{2}, X_{3}\right)-G\left(X_{1}, X_{2}\right) . F \circ \Phi=$ $H\left(Z, Z^{p} w, Z^{q} w, U(Z, w)\right)-G\left(Z, Z^{p} w\right)$. Let $Z_{1}=Z, Z_{2}=Z^{p} w$. Thus on $\Omega \subset$ $\mathbf{C}^{2} \backslash\left\{Z_{1}=0\right\}, \quad F \circ \Phi=H\left(Z_{1}, Z_{2}, Z_{1}^{q-p} Z_{2} U\left(Z_{1}, Z_{2} / Z_{1}^{p}\right)\right)-G\left(Z_{1}, Z_{2}\right) \equiv 0$. Thus $F \circ \Phi \equiv 0$ by analytic continuation. Therefore $\operatorname{cg}\left(\operatorname{ker} \Phi^{*}\right) \neq 0$.

$(\Rightarrow)$ Suppose $F \in \mathbf{C}_{3}, F \neq 0$, and $F \in \operatorname{ker} \Phi^{*}$. We must have $\partial F / \partial X_{3} \neq 0$ because the homomorphism from $\mathbf{C}_{2}$ to $\mathbf{C}_{2}$ given by $f \rightarrow f\left(Z, Z^{p} w\right)$ is injective. Suppose $F=\sum_{i=0}^{\infty} P_{i}\left(X_{1}, X_{2}\right) X_{3}^{i}, P_{i} \in \mathrm{C}_{2}$ for each $i$ and $P_{i_{0}} \neq 0$ for some $i_{0} \geqslant 1$. Let $H \in \mathbf{C}_{3}$ be given by $H=\sum_{i=1}^{\infty} P_{i}\left(X_{1}, X_{2}\right) X_{3}^{i}$. Thus $H\left(Z, Z^{p} w, Z^{q} w U(Z, w)\right)=-P_{0}\left(Z, Z^{p} w\right)$. Letting $Z_{1} \equiv Z, Z_{2} \equiv Z^{p} w$ yields $H\left(Z_{1}, Z_{2}, Z_{1}^{q-p} Z_{2} U\left(Z_{1}, Z_{2} / Z_{1}^{p}\right)\right)=-P_{0}\left(Z_{1}, Z_{2}\right)$ $\in \mathbf{C}_{2}$.

REMARK 1.13. (1) From the proof we see the existence of $H$ in Lemma 1.11 is equivalent to the existence of $H \in \mathrm{C}_{3}$ such that $H\left(Z, Z^{p} w, Z^{q} w U(Z, w)\right) \in$ $\mathrm{C} \ll Z, Z^{p} w \gg$, the ring of convergent power series in $Z$ and $Z^{p} w$.

(2) In Lemma 1.11 it is only necessary to show the existence of a formal power series $H$ such that $X_{3} \mid H, H \neq 0$, and $H\left(Z_{1}, Z_{2}, Z_{1}^{q-p} Z_{2} U\left(Z_{1}, Z_{2} / Z_{1}^{p}\right)\right)$ is a formal power series in $Z_{1}$ and $Z_{2}$ to imply $\operatorname{cg}\left(\operatorname{ker} \Phi^{*}\right)=1$. This follows from [Gab] for $n=3$. 
2. In this section we provide several consequences of Lemma 1.11. We assume $\Phi$ is of the form (1.10) and $U$ is the power series appearing in (1.10).

Proposition 2.1. If $U$ is rational in $w$ then $\operatorname{cg}\left(\operatorname{ker} \Phi^{*}\right)=1$.

Proof. Let $U(Z, w)=P(Z, w) / Q(Z, w)$ where $P(Z, w)=\sum_{i=1}^{N} P_{i}(Z) w^{i}, Q(Z, w)$ $=\sum_{i=1}^{M} Q_{i}(Z) w^{i}$ with $P_{i}, Q_{i} \in \mathbf{C}_{1}$ for all $i$. Thus

$$
U\left(Z_{1}, Z_{2} / Z_{1}^{p}\right)=\left(\sum_{i=1}^{N} P_{i}\left(Z_{1}\right)\left(Z_{2} / Z_{1}^{p}\right)^{i}\right) /\left(\sum_{i=1}^{M} Q_{i}\left(Z_{1}\right)\left(Z_{2} / Z_{1}^{p}\right)^{i}\right) .
$$

Multiplying the numerator and denominator of (2.2) by $Z_{\mathrm{l}}^{(N+M) p}$ clears each of its $1 / Z_{1}$ dependence. Thus $H \equiv \sum_{i=0}^{M} Q_{i}\left(Z_{1}\right) Z_{2}^{i} Z_{1}^{(N+M-i) p} Z_{3}$ works in Lemma 1.11.

REMARK 2.3. (1) Proposition 2.1 proves the final conjecture of [H-2]. That is, if $U$ is a polynomial in $w$, then $\operatorname{cg}\left(\operatorname{ker} \Phi^{*}\right)=1$.

(2) Proposition 2.1 illustrates the sense in which Lemma 1.11 is a generalization of the standard algebraic geometry technique of going to projective space, solving the appropriate equations and then homogenizing to get back to the original setting.

We further illustrate Remark 2.3(2) with some examples.

EXAMPLe 2.4. $\Phi=\left(Z, Z w, Z w\left(1+w+\cdots+w^{N}\right)\right)$.

In homogeneous coordinates we have $\Phi=\left(1, Z_{2}, Z_{2}\left(1+Z_{2}+\cdots+Z_{2}^{N}\right)\right)$ and the relation is $Z_{3}=Z_{2}\left(1+Z_{2}+\cdots+Z_{2}^{N}\right)$. Homogenizing yields

$$
Z_{1}^{N} Z_{3}=Z_{2}\left(Z_{1}^{N}+Z_{1}^{N-1} Z_{2}+\cdots+Z_{2}^{N}\right) \text {. }
$$

Thus $H \equiv Z_{1}^{N} Z_{3}$ works and $Z_{1}^{N} Z_{3}-Z_{2}\left(Z_{1}^{N}+Z_{1}^{N-1} Z_{2}+\cdots+Z_{2}^{N}\right) \in \operatorname{ker} \Phi^{*}$.

EXAmple 2.5. $\Phi=\left(Z, Z w, Z w\left(1+\sum_{i=1}^{N} P_{i}(Z) w^{i}\right)\right)$.

In Example 2.5 we cannot consider homogeneous coordinates as in Example 2.4; however, the same approach leads to

$$
Z_{1}^{N} U\left(Z_{1}, Z_{2} / Z_{1}\right)=Z_{1}^{N}+Z_{1}^{N-1} P_{1}\left(Z_{1}\right) Z_{2}+\cdots+P_{N}\left(Z_{1}\right) Z_{2}^{N} .
$$

That is, $H=Z_{1}^{N} Z_{3}$ works and

$$
Z_{1}^{N} Z_{3}-Z_{2}\left[Z_{1}^{N}+Z_{1}^{N-1} P_{1}\left(Z_{1}\right) Z_{2}+\cdots+P_{N}\left(Z_{1}\right) Z_{2}^{N}\right] \in \operatorname{ker} \Phi^{*} .
$$

EXAMPLE 2.6. $\Phi=\left(Z, Z w, Z w \sum_{i=0}^{\infty} w^{i}\right)$.

Here $U\left(Z_{1}, Z_{2} / Z_{1}\right)=Z_{1} /\left(Z_{1}-Z_{2}\right)$. So $H \equiv\left(Z_{1}-Z_{2}\right) Z_{3}$ works and

$$
\left(Z_{1}-Z_{2}\right) Z_{3}-Z_{2} Z_{1} \in \operatorname{ker} \Phi^{*} \text {. }
$$

Example 2.6 serves as motivation for another consequence of Lemma 1.11; namely,

Proposition 2.7. If $U\left(Z_{1}, Z_{2} / Z\right.$ p) is meromorphic then $\operatorname{cg}\left(\operatorname{ker} \Phi^{*}\right)=1$.

Proof. By "meromorphic" we mean there exists $F$ and $G$ in $\mathbf{C}_{2}$ so that $U\left(Z_{1}, Z_{2} / Z_{1}^{p}\right) \equiv G\left(Z_{1}, Z_{2}\right) / F\left(Z_{1}, Z_{2}\right)$ off the zero set of $F$ near 0 . As convergent power series in $Z_{1}, Z_{2}$, and $1 / Z_{1}$ this means $U\left(Z_{1}, Z_{2} / Z_{1}^{p}\right) \equiv G\left(Z_{1}, Z_{2}\right) / F\left(Z_{1}, Z_{2}\right)$. Clearly $H \equiv F\left(X_{1}, X_{2}\right) X_{3}$ works in Lemma 1.11 .

The converse of Proposition 2.7 is not true as shown by

EXAmple 2.8. $\Phi=(Z, Z w, Z w \sqrt{1-w})$. 
In this example $U\left(Z_{1}, Z_{2} / Z_{1}\right)=\sqrt{1-Z_{2} / Z_{1}}$ is not meromorphic. However $\operatorname{cg}\left(\operatorname{ker} \Phi^{*}\right)=1$ because $Z_{3}^{2} Z_{1}-Z_{2}\left(Z_{1}-Z_{2}\right) \in \operatorname{ker} \Phi^{*}$.

Example 2.8 motivates the following

Proposition 2.9. If $U(Z, w)$ is algebraic in $w$ then $\operatorname{cg}\left(\operatorname{ker} \Phi^{*}\right)=1$.

Proof. Suppose there exists $P \in \mathrm{C}_{3}$ of the form $P=\sum_{j=0}^{N} \alpha_{j}\left(X_{1}, X_{2}\right) X_{3}^{i}$ where for each $j, \alpha_{j}\left(X_{1}, X_{2}\right) \in \mathbf{C}\left\langle X_{1}\right\rangle\left[X_{2}\right]$, polynomials in $X_{2}$ with coefficients being convergent power series in $X_{1}$, such that $P(Z, w, U(Z, w)) \equiv 0$ but $P \neq 0$. On $\mathbf{C}_{2} \backslash\{Z=0\}$ we thus have

$$
0 \equiv \sum_{j=0}^{N} \alpha_{j}\left(Z, w / Z^{p}\right) U^{j}\left(Z, w / Z^{p}\right) .
$$

Relabel the variables via $Z \rightarrow Z_{1}$ and $w \rightarrow Z_{2}$. Let

$$
M=\max \left\{\text { degree in } Z_{2} \text { of } \alpha_{j}\left(Z_{1}, Z_{2}\right): 1 \leqslant j \leqslant N\right\} .
$$

Multiplying (2.10) by $Z_{1}^{M p} Z_{1}^{(q-p) N} Z_{2}^{N}$ yields

$$
0 \equiv \sum_{j=0}^{N} Z_{1}^{M p} \alpha_{j}\left(Z_{1}, Z_{2} / Z_{1}^{p}\right) Z_{1}^{(N-j)(q-p)} Z_{2}^{N-j}\left[Z_{1}^{q-p} Z_{2} U\left(Z_{1}, Z_{2} / Z_{1}^{p}\right)\right]^{j} .
$$

Hence let $H \equiv \sum_{j=1}^{N} b_{j}\left(X_{1}, X_{2}\right) X_{3}^{j}$ where

$$
b_{j}\left(X_{1}, X_{2}\right) \equiv Z_{1}^{M p} \alpha_{j}\left(Z_{1}, Z_{2} / Z_{1}^{p}\right) Z_{1}^{(N-j)(q-p)} Z_{2}^{N-j} .
$$

$H$ is the desired member of $\mathbf{C}_{3}$ for use in Lemma 1.11 .

In order to better understand the relevance of Example 0.2, we need

Proposition 2.11. Let $\Phi \equiv\left(Z, Z^{p} w, Z^{q} w U(Z, w)\right)$ be in form (1.10) and let $\Psi \equiv(Z, Z w, Z w U(Z, w))$. Then $\operatorname{cg}\left(\operatorname{ker} \Phi^{*}\right)=1$ implies $\operatorname{cg}\left(\operatorname{ker} \Psi^{*}\right)=1$.

Proof. Define $\Gamma: \mathbf{C}^{3} \rightarrow \mathbf{C}^{3}$ by $\Gamma\left(X_{1}, X_{2}, X_{3}\right) \equiv\left(X_{1}, X_{1}^{p^{-1}} X_{2}, X_{1}^{q-1} X_{3}\right)$. Then $\Gamma^{*}$ : $C_{3} \rightarrow C_{3}$ is injective and $\Phi^{*}=\Psi^{*} \circ \Gamma^{*}$. The conclusion follows immediately.

We can now prove

THEOREM 2.12. Suppose $M^{2} \subset \mathrm{C}^{3}$ is a real-analytic two-dimensional submanifold of $\mathrm{C}^{3}$ and the associated holomorphic mapping $\Phi: \mathbf{C}^{2} \rightarrow \mathbf{C}^{3}$ has been reduced to the form of (1.10). Further suppose $U$ in (1.10) is independent of $Z . M^{2}$ is not a local uniqueness set for holomorphic functions of $\mathbf{C}^{3}$ if and only if $U$ is algebraic.

Proof. $(\Leftarrow)$ This follows from Propositions 2.9 and 1.2.

$(\Rightarrow)$ Suppose $\operatorname{cg}\left(\operatorname{ker} \Phi^{*}\right)=1$. By Proposition $2.11 \operatorname{cg}\left(\operatorname{ker} \Psi^{*}\right)=1$. Let $f \in \operatorname{ker} \Psi^{*}$ such that $f \equiv \sum_{i=0}^{\infty} P_{i}\left(X_{1}, X_{2}, X_{3}\right) \neq 0$ for homogeneous polynomials $P_{i}$ of degree $i$. Then

$$
0 \equiv f \circ \Psi=\sum_{i=0}^{\infty} P_{i}(Z, Z w, Z w U(w))=\sum_{i=0}^{\infty} P_{i}(1, w, U(w)) Z^{i} .
$$

Therefore, $P_{i}(1, w, U(w)) \equiv 0$ for all $i$ and hence for some $i_{0}$ with $P_{i_{0}} \not 0$.

REMARK 2.13. From Theorem 2.12 we see the Osgood example as presented in [G-R, p. 121] is the "only" way to construct $\Phi: \mathbf{C}^{2} \rightarrow \mathbf{C}^{3}$ with $\Phi^{*}$ being injective and 
$U$ being independent of $Z$. This means that Example 0.2 is the "only" way to construct a real-analytic two-dimensional submanifold of $\mathbf{C}^{3}$ which has no transcendental dependence on $X$ and is a local uniqueness set for $\mathbf{C}^{3}$ at 0 .

Because of Remark 2.13, we consider the situation $\Phi=\left(Z, Z^{p} w, Z^{q} w U(Z, w)\right)$ where $\partial U / \partial Z Z 0$. In general $\operatorname{cg}\left(\operatorname{ker} \Phi^{*}\right)=1$ is not sufficient to yield $U(Z, w)$ algebraic in $w$. Consider

EXAMPLE 2.14. $\Phi \equiv(Z, Z w, Z w \exp (Z w))$.

Obviously, $f\left(X_{1}, X_{2}, X_{3}\right) \equiv X_{3}-X_{2} e^{X_{2}} \in \operatorname{ker} \Phi^{*}$ but $U$ is not algebraic in $w$. However, we can further simplify the form of $\Phi$ in (1.10) by subtracting off any terms of $Z^{q} w(U(Z, w)-1)$ which are monomials in $Z$ and $Z^{p} w$, a range coordinate change. We are left with

$$
\Phi=\left(Z, Z^{p} w, Z^{q} w U(Z, w)\right)
$$

where

$$
U=1+\sum_{n=1}^{\infty} \sum_{m=0}^{p(n+1)-q-1} \alpha_{m, n} Z^{m} w^{n}
$$

In particular, Example 2.14 simplifies to $\Phi=(Z, Z w, Z w)$ for which the problem is trivial. It remains to consider examples like

EXAmple 2.17. $\Phi=\left(Z, Z w, Z w \exp \left(Z w^{2}\right)\right)$.

Example 2.17 is in the form of (2.15) with $U=\exp \left(Z w^{2}\right)$ in the form of (2.16). Because of the $z$ dependence of $U$ we are not able to use an algebraic argument like that in the necessity proof of Theorem 2.12 to conclude $\operatorname{cg}\left(\operatorname{ker} \Phi^{*}\right)=0$. However, for this example we may appeal to the following geometric argument to conclude $\operatorname{cg}\left(\operatorname{ker} \Phi^{*}\right)=0$. Suppose $F \in \mathbf{C}_{3}, F \neq 0$ and $F \in \operatorname{ker} \Phi^{*}$. Without loss of generality we may assume $F$ is irreducible. Let $W$ denote the germ at 0 of $\left\{z \in \mathbf{C}^{3}: F(z)=0\right\}$ and $V$ denote $\left\{\left(X_{1}, X_{2}, X_{2} \exp \left(X_{2}^{2} / X_{1}\right)\right): 0<\left|X_{1}\right|<r\right.$ and $\left.\left|X_{2}\right|<r\right\}$ for some appropriately chosen $r>0$ depending on $F$. For fixed $X_{2}$ let $V_{X_{2}}$ denote $\left\{\left(X_{1}, X_{2}, X_{2} \exp \left(X_{2}^{2} / X_{1}\right): 0<\left|X_{1}\right|<r\right\}\right.$. For every $X_{2}$ with $\left|X_{2}\right|<r$ we have $\overline{V_{X_{2}}} \subset$ $W$. The essential nature of the singularity at $X_{1}=0$ yields $\overline{V_{X_{2}}}=\{0\} \times\left\{X_{2}\right\} \times \mathbf{C}_{0}$, where $\mathbf{C}_{0}$ denotes the germ of $\mathbf{C}$ at 0 . Letting $\left|X_{2}\right| \rightarrow 0$ yields $\{0\} \times \mathbf{C}_{0}^{2} \subset W$. Hence $F\left(Z_{1}, Z_{2}, Z_{3}\right)=Z_{1} Q\left(Z_{1}, Z_{2}, Z_{3}\right)$ for some unit $Q$ in $\mathbf{C}_{3}$. This implies that $Z_{1}$ vanishes on $V \subset W$, a contradiction.

However we may still have $U$, expressed in the form of (2.16), not algebraic in $w$ but $\operatorname{cg}\left(\operatorname{ker} \Phi^{*}\right)=1$. Consider

EXAMPLe 2.18. $\Phi=(Z, Z w, Z w[1 /(1-\exp (Z w) w)])$.

In Example 2.18, $U(Z, w)$ is not algebraic in $w$ but

$$
f\left(X_{1}, X_{2}, X_{3}\right) \equiv X_{1} X_{2}-X_{3}\left(X_{1}-X_{2} e^{X_{2}}\right) \in \operatorname{ker} \Phi^{*} .
$$

We are thus led to a further "refinement" in the form of $U$. From (2.16) we see

$$
U\left(X_{1}, X_{2} / X_{1}^{p}\right)=1+\sum_{n=1}^{\infty} g_{n}\left(X_{2}\right) X_{1}^{-n}
$$


where each $g_{n} \in \mathbf{C}_{1}$ and (2.19) is valid (as a function) on the open set $\left\{\left(X_{1}, X_{2}\right) \in\right.$ $\left.\mathrm{C}^{2}\left|\left(\left|X_{2}\right| / r\right)^{1 / p}<\right| X_{1} \mid<r\right\}$ for some $r>0$. For notational convenience we introduce new variables $\tau_{1}, \tau_{2}$ and let $\tilde{U}\left(\tau_{1}, \tau_{2}\right)$ denote the formal power series defined by

$$
\tilde{U}\left(\tau_{1}, \tau_{2}\right) \equiv 1+\sum_{n=1}^{\infty} g_{n}\left(\tau_{1}\right) \tau_{2}^{n}
$$

where each $g_{n} \in \mathbf{C}_{1}$ comes from (2.19). We also let $\tilde{U}\left(\tau_{1}, \tau_{2}\right)$ denote the function represented by (2.20) on the set $\Omega \equiv\left\{\left(\tau_{1}, \tau_{2}\right) \in \mathbf{C}^{2}\left|\left(r /\left|\tau_{1}\right|\right)^{1 / p}>\right| \tau_{2} \mid>1 / r\right\}$. By the same argument used to prove Proposition 2.9 we can prove

Proposition 2.21. If $\tilde{U}\left(\tau_{1}, \tau_{2}\right)$ is algebraic in $\tau_{2}$ then $\operatorname{cg}\left(\operatorname{ker} \Phi^{*}\right)=1$.

By Proposition 2.11 and the geometric argument following Example 2.17 we can now prove

Proposition 2.22. Suppose $\tilde{U}$ in (2.20) is convergent on $\left\{\left|\tau_{1}\right|<r\right\} \times \mathbf{C}$ for some $r>0$. If $\infty$ is an essential singularity for the function $\tau_{2} \mapsto \tilde{U}\left(\tau_{1}, \tau_{2}\right)$ for each fixed $\tau_{1} \neq 0$ with $\left|\tau_{1}\right|$ small, then $\operatorname{cg}\left(\operatorname{ker} \Phi^{*}\right)=0$.

Propositions 2.21 and 2.22 can be combined with Proposition 1.2 to prove

THEOREM 2.23. Suppose $M^{2}$ is a two-dimensional real-analytic submanifold of $\mathbf{C}^{3}$ and the associated holomorphic mapping $\Phi: \mathbf{C}^{2} \rightarrow \mathbf{C}^{3}$ has been reduced to the form of (2.15) with $U$ in the form of (2.19) and $\tilde{U}$ as defined in (2.20). Further, suppose for some $r>0, \tilde{U}\left(\tau_{1}, \tau_{2}\right)$ is convergent on $\left\{\left|\tau_{1}\right|<r\right\} \times \mathbf{C}, M^{2}$ is not a local uniqueness set for holomorphic functions of $\mathbf{C}^{3}$ if and only if $\tilde{U}\left(\tau_{1}, \tau_{2}\right)$ is algebraic in $\tau_{2}$.

We now consider the case for which $\tilde{U}\left(\tau_{1}, \tau_{2}\right)$ in $(2.20)$ is a convergent power series with finite biradius of convergence. We may, without loss of generality, assume the biradius is $(1,1)$. For each fixed $\tau_{1}$ with $\left|\tau_{1}\right|<1$ let $U_{\tau_{1}}$ denote the function given by $\tau_{2} \mapsto \tilde{U}\left(\tau_{1}, \tau_{2}\right)$ for $\left|\tau_{2}\right|<1$. For each small $\varepsilon>0$ let $A(\varepsilon) \equiv\left\{\tau_{2} \in\right.$ C $\left.|1-\varepsilon<| \tau_{2} \mid<1\right\}$. The geometric argument following Example 2.17 can be applied to yield

Proposition 2.24. If $\overline{U_{\tau_{1}}(A(\varepsilon))}=\mathrm{C}$ for all sufficiently small $\varepsilon>0$ and a dense set of $\tau_{1}$ near 0 , then $\operatorname{cg}\left(\operatorname{ker} \Phi^{*}\right)=0$.

REMARK 2.25. For the most general case of a real-analytic two-dimensional submanifold, $M^{2}$ of $\mathrm{C}^{3}$, the question of whether $M^{2}$ is a local uniqueness set for holomorphic functions of $\mathbf{C}^{3}$ now involves two-dimensional Nevanlinna theory applied to the function $\tilde{U}\left(\tau_{1}, \tau_{2}\right)$ in (2.20). For example, if for each fixed $\tau_{1} \neq 0$ the function $U_{\tau_{1}}$ defined above is of unbounded characteristic then $M^{2}$ is a local uniqueness set for holomorphic functions of $\mathbf{C}^{3}$ (see [C-L, p. 67]). Applying results from Nevanlinna theory one can conclude several such technical results, however they do not appear to significantly contribute to our understanding of the original problem and will not be included.

We conclude with a conjecture which is motivated by the above examples and our unsuccessful attempts to construct a counterexample. 
Conjecture. The converse of Proposition 2.21 is also true. That is, if $M$ is not a uniqueness set for $\mathbf{C}^{3}$ then $\tilde{U}\left(\tau_{1}, \tau_{2}\right)$ in (2.20) depends algebraically on $\tau_{2}$.

\section{REFERENCES}

[C-L] E. F. Collingwood and A. T. Lohwater, The theory of cluster sets, Cambridge Univ. Press, London, 1966.

[G-R] H. Grauert and R. Remmert, Analytische Stellenalgebren, Springer-Verlag, New York, 1970.

[Gab] A. M. Gabrielov, Formal relations between analytic functions, Izv. Akad. Nauk SSSR Ser. Mat. 37 (1973), 1056-1088.

[H-1] G. A. Harris, The traces of holomorphic functions on real submanifolds, Trans. Amer. Math. Soc. 242 (1978), 205-223.

[H-2] _ Geometry near a C. R. singularity, Illinois J. Math. 25 (1981), 147-158.

[M] P. Milman, Analytic and polynomial homomorphisms of analytic rings, Math. Ann. 232 (1978), $247-253$.

Department of Mathematics, TeXas Tech University, LubBock, TeXas 79409 